\title{
A Measurement System for the Complex Far-Field of Physically Large Antenna Arrays Under Noisy Conditions Utilizing the Equivalent Electric Current Method
}

\author{
Tore Lindgren, Member, IEEE, Jonas Ekman, Member, IEEE, and Staffan Backén, Student Member, IEEE
}

\begin{abstract}
Precipitation in the form of snow or rain could severely degrade the performance of large antenna arrays, in particular if knowledge about the beam shape and pointing direction in absolute numbers is necessary. In this paper, a method of estimating the far-field of each individual antenna element using the equivalent electric current approach is presented. Both a least squares estimator and a Kalman filter was used to solve the resulting system of equation and their performance was compared. Simulation results shows that the estimated far-field for one antenna element is very accurate if there is no noise on the signal. During noisier conditions the Kalman filter gives less noisy results while the systematic errors are slightly larger compared to the least squares estimator.
\end{abstract}

Index Terms-Antenna arrays, antenna measurements.

\section{INTRODUCTION}

$\mathbf{L}$ ARGE antenna arrays operating in an arctic environment may have their performance significantly degraded as the properties of the individual antenna elements change due to weather effects, in particular snowfall [1]. This could cause severe problems in applications where knowledge about the gain and pointing direction of the main beam of the antenna array is needed in absolute numbers. Good knowledge about each antenna element's active radiation pattern can significantly improve the performance of the beamforming process. In this paper, the method of calculating equivalent electric current in order to estimate the far-field of the antennas [2], [3] is used and adapted to the situation when the antenna array consists of relatively simple antenna elements (e.g., dipoles or yagi-antennas) but where the whole antenna array is physically and electrically large.

The antenna array considered here is the planned EISCAT_3D incoherent scatter radar [4] which is an upgrade of the existing tristatic EISCAT UHF radar in northern Scandinavia. The radar system will consist of one main transmit/receive site and multiple receive-only sites at 90-280 km from the main site. Each

Manuscript received March 13, 2009; revised February 10, 2010; accepted April 12, 2010. Date of publication July 01, 2010; date of current version October 06, 2010.

The authors are with the Department of Computer Science and Electrical Engineering, Luleå University of Technology, Luleå 97187, Sweden (e-mail: tore.lindgren@ltu.se).

Digital Object Identifier 10.1109/TAP.2010.2055780 site will have an antenna array consisting of up to 16000 antenna elements operating in the $210-240 \mathrm{MHz}$ frequency band. Since the ESCAT_3D radar will be a multistatic radar with a very narrow beam, the pointing direction of the beam must be known with good accuracy. The maximum allowed timing error between any two antenna elements in the array has been found to be $160 \mathrm{ps}$ [5]. The timing error is composed of jitter in the local oscillator and in the analog-to-digital converter (ADC), errors introduced in the clock distribution system, and changes in the phase of the far-field pattern of the antenna elements [6]. Since the radar will operate continuously the far-field of the antenna elements will need to be measured regularly. In particular, the measurement system must be able to detect any changes in the phase characteristics. The following limiting factors of the measurement system have been identified due to the specific application of ionospheric radar and the physical and electrical size of the system as follows.

- The signals of interest to the users of the system are very weak [7]. The probes used for the measurements should therefore be located outside the field-of-view of the antenna array, as much as possible, in order to minimize interference and diffraction effects that could degrade the performance of the radar.

- The beamforming of the antenna array will be digital with each antenna element sampled individually. With up to 16000 antenna elements it is essential to keep the calculations to a minimum. Thus, the number of probes used for the measurements should be minimized.

- It is not practical to place the probes in the far-field of the antenna array due to the physical size of the antenna. Hence, the calibration system must be able to accurately calculate the phase of the far-field with probes located at varying distances from the antenna elements.

As a result of the limiting factors mentioned above some of the conventional measurement methods may not be suitable for the type of antenna system considered in this paper. The far-field pattern of large aperture antennas is traditionally measured using radio sources such as quasars or distant galaxies [8]. With this approach it is possible to get accurate gain and phase characteristics of the antenna. The typical signal strength is, however, too low to be detected by the individual antenna elements in an array. A similar technique, described in [9], uses spacecrafts as receivers for signals transmitted by the Antenna Under Test (AUT). To apply this to the EISCAT_3D radar, the 
spacecraft would need to act as the transmitter since not all sites will have transmitting capabilities. A major drawback of such a calibration system is that it is unlikely that a spacecraft can be dedicated to the radar and the calibration of the radar would therefore rely on signals transmitted for other purposes (signals-of-opportunity). Although the measurement methods discussed above are not suitable as a sole calibration system they could be a valuable complement to a measurement system located in the near-field of the antenna array.

The far-field of an antenna can also be estimated using measurements in the near-field. This typically requires more calculations than the far-field techniques. A comprehensive overview of near-field measurement techniques can be found in [10]. The methods described assume that all measured points lie on the same surface (planar, cylindrical, or spherical) within a fraction of a wavelength. This may not be possible to achieve in the EISCAT_3D radar due to the size of the system. The method described in this paper is instead based on the equivalent current approach where the antenna is replaced by an equivalent electric and/or magnetic current, which is described in [2]. A clear advantage is that the equivalent current approach is less sensitive to non-ideal probe positions than traditional near-field measurement methods. It was shown in [11] that the current measurement technique gives accurate results when estimating the far-field of large antenna arrays.

In this paper a measurement system using the equivalent electric current method for the EISCAT_3D antenna array is described. To reduce the size of the matrices in the system of equations to be solved, the near-field of each antenna element is measured separately, with the probes used as test transmitters. This is possible since all elements will have a separate front-end. The method presented has been simulated in order to illustrate the performance of the approach.

\section{The MEASUREd EleCtric FIELD}

\section{A. The Electric Field Due to the Current Distribution on One}

\section{Antenna}

The electric field $\mathbf{E}$ at a point $\mathbf{r}$ in the near-field of an antenna consisting of a perfect electric conductor can be calculated using

$$
\mathbf{E}(\mathbf{r})=-j \omega \mathbf{A}(\mathbf{r})-j \frac{1}{\omega \mu \varepsilon} \nabla(\nabla \cdot \mathbf{A}(\mathbf{r}))
$$

with

$$
\mathbf{A}(\mathbf{r})=\mu \int_{S^{\prime}} \frac{e^{-j \omega R}}{4 \pi R} \mathbf{J}\left(\mathbf{r}^{\prime}\right) d S^{\prime}
$$

where $\omega$ is the angular frequency, $\mu$ and $\varepsilon$ is the permeability and the permittivity of the medium, $S^{\prime}$ is the surface of the antenna, $\mathbf{J}\left(\mathbf{r}^{\prime}\right)$ is the electric current at the point $\mathbf{r}^{\prime}$ on the antenna, and $R=\left|\mathbf{r}-\mathbf{r}^{\prime}\right|$.

The current distribution can be expanded using basis functions according to

$$
\mathbf{J}\left(\mathbf{r}^{\prime}\right)=\sum_{n=1}^{N} \alpha_{n} \mathbf{f}_{n}\left(\mathbf{r}^{\prime}\right)
$$

where $\alpha_{n}$ are constants and $\mathbf{f}_{n}\left(\mathbf{r}^{\prime}\right)$ are suitable basis functions. The near-field integral (1) can then be written as

$$
\mathbf{E}(\mathbf{r})=\sum_{n=1}^{N} \alpha_{n} \mathbf{G}_{n}(\mathbf{r})
$$

with

$$
\mathbf{G}_{n}=-j \omega \mathbf{A}_{n}(\mathbf{r})-j \frac{1}{\omega \mu \varepsilon} \nabla\left(\nabla \cdot \mathbf{A}_{n}(\mathbf{r})\right)
$$

and

$$
\mathbf{A}_{n}(\mathbf{r})=\mu \int_{S^{\prime}} \frac{e^{-j \omega R}}{4 \pi R} \mathbf{f}_{n}\left(\mathbf{r}^{\prime}\right) d S^{\prime}
$$

The field measured at the point $\mathbf{r}$ will be affected by the polarization properties of the probes. Therefore,

$$
E(\mathbf{r})=\mathbf{E}(\mathbf{r}) \cdot \hat{\mathbf{p}}^{*}=\sum_{n=1}^{N} \alpha_{n} \mathbf{G}_{n} \cdot \hat{\mathbf{p}}^{*}
$$

where $\hat{\mathbf{p}}^{*}$ is the complex conjugate of the polarization vector $\hat{\mathbf{p}}$ which describes the polarization of the probe.

\section{B. The Difference Between Two Antennas in an Antenna Array}

The expressions above relates the current distribution, described using basis functions, to the electric near-field. Of interest when implementing the beamforming algorithms is the difference in the amplitude and phase of the far-field between two antenna elements, as this will affect the direction of the beam. Also, calculating this difference enables the use of signals-of-opportunity, where the absolute phase is not known, in the estimation process. One antenna element could then be temperature controlled (i.e., free from snow) and used as a reference element. To calculate the difference in the measured electric field between two antenna elements, $p$ and $q$, (7) is rewritten as

$$
E^{(q)}(\mathbf{r})-E^{(p)}(\mathbf{r})=\sum_{n=1}^{N}\left(\alpha_{n}^{(q)} \mathbf{G}_{n}^{(q)}-\alpha_{n}^{(p)} \mathbf{G}_{n}^{(p)}\right) \cdot \hat{\mathbf{p}}^{*} .
$$

Assuming that $p$ is the reference element and $q$ is the AUT, the constants $\alpha_{n}^{(q)}$ can be written as

$$
\alpha_{n}^{(q)}=\alpha_{n}^{(p)}+\delta_{n}
$$

where $\delta_{n}$ is the difference between $\alpha_{n}^{(p)}$ and $\alpha_{n}^{(q)}$. This makes it possible to write (8) as

$$
\Delta E=\sum_{n=1}^{N}\left(\alpha_{n}^{(p)}\left(\mathbf{G}_{n}^{(q)}-\mathbf{G}_{n}^{(p)}\right)+\delta_{n} \mathbf{G}_{n}^{(q)}\right) \cdot \hat{\mathbf{p}}^{*}
$$

where $\Delta E=E^{(q)}(\mathbf{r})-E^{(p)}(\mathbf{r})$. If the reference element is assumed to be unaffected by the environment the term $\alpha_{n}^{(p)}\left(\mathbf{G}_{n}^{(q)}-\right.$ $\mathbf{G}_{n}^{(p)}$ ) will be known, with $\mathbf{G}_{n}^{(p)}$ and $\mathbf{G}_{n}^{(q)}$ given by (5). The only remaining unknowns are then the constants $\delta_{n}$. Using matrix notation (10) is rewritten as

$$
G \delta=\Delta E-\left(G-G^{(p)}\right) \alpha^{(p)}
$$

where

$$
G=\mathbf{G}^{(q)} \cdot \hat{\mathbf{p}}^{*} .
$$




\section{ESTIMATING THE CURRENT DistriBUTION ON THE ANTENNAS}

There are several methods that can be used to solve the system of equations in (11). In the original work on the equivalent current technique by Petre and Sarkar [2] the conjugate gradient (CG) method was used [13]. This method results in a stable solution and it is also computationally efficient, which is crucial when large matrices are involved, such as when aperture antennas are analyzed. In the present paper the antenna elements in the array are crossed Yagi-antennas, which can be modeled using a relatively low number of unknowns. Thus, the number of probes used for the measurements are kept to a minimum, which also reduces the computational load.

The performance of the measurement system is in this paper evaluated under noisy conditions. The observed field strength is modeled as

$$
\Delta E_{\text {observed }}=\Delta E_{\text {true }}+\mathbf{w}
$$

where $\mathbf{w}$ is the measurement noise. The noise is assumed to be zero mean and uncorrelated between measurements. The coefficients $\alpha^{p}$ of the reference element are assumed to be known a priori since the reference element is unaffected by the environment. Including this into the equation does therefore not affect the properties of the noise. The system of equations can thus be written as

$$
G \delta=b
$$

with

$$
b=\Delta E-\left(G-G^{(p)}\right) \alpha^{(p)}+\mathbf{w} .
$$

Two fundamentally different approaches of estimating $\delta$ are classical and Bayesian estimation [14]. In the classical approach, the parameters of interest are assumed to be deterministic but unknown. The Bayesian approach, on the other hand, assumes some prior knowledge about the properties of the parameters, such as the dynamic behavior of the system. This knowledge can then be used to improve the estimation accuracy. As a classical method the least squares (LS) estimator is chosen and the Kalman filter is chosen as a Bayesian estimator. Both these methods are used extensively in the fields of signal processing and control theory and are under certain conditions optimal (see the following two subsections). It should however be noted that other methods may be more suitable in an implementation where the properties of the signal are affected by hardware.

The errors in the estimated radiation pattern of the antenna elements will consist of a random- and a systematic part. For a small antenna array the random part could be expected to have the largest influence on the beam-forming if the systematic error is small. This might not be the case for large antenna arrays, where the random error will be averaged over the array. In this case a small systematic error could have more severe consequences on the beam-forming than random variations over the array. This need to be kept in mind when comparing the results from the LS estimator with the Kalman filter.

\section{A. Least Squares}

In the LS estimator the square error is minimized using

$$
\delta=\left(G^{H} G\right)^{-1} G^{H} b
$$

where $G^{H}$ is the hermitian transpose of $G$. The error in $\delta$ will be zero on average if the measurements $b$ are composed of a deterministic signal and a zero mean noise, see chapter 6 in [14]. Further, it is the optimal minimum variance unbiased (MVU) estimator if the error is white Gaussian noise (with zero mean). This is assumed in the simulations presented in the next section but will not be the case in an implementation where the white noise is filtered and thus no longer completely white. Other estimators may be more appropriate, the LS estimator is nevertheless usable even in this case since the error will be zero on average.

\section{B. Kalman Filter}

Since it is necessary to measure the performance of each antenna element continuously, a Kalman filter can be used to reduce the errors induced by noisy measurements, see chapter 13 in [14]. The Kalman filter has the advantage over the LS estimator that it works even if the noise are non-stationary since the properties of the noise is estimated in the filter. Also, the Kalman filter is a sequential minimum mean square error (MMSE) estimator. Thus, it may not provide a better estimate of the parameters in all cases but, given a system such as described in this paper, the estimate will be better on average.

The performance of the AUT relative to the reference antenna at a given time, $t_{k}$, is in this case described by the state

$$
\mathbf{x}\left(t_{k}\right)=\left[\begin{array}{l}
\delta\left(t_{k}\right) \\
\dot{\delta}\left(t_{k}\right)
\end{array}\right]
$$

where $\delta\left(t_{k}\right)$ are the coefficients describing the difference in the current distribution on the AUT and the reference antenna element at time $t_{k}$, from (14), and $\dot{\delta}\left(t_{k}\right)$ are the time derivatives of these coefficients. Assuming that there is a linear change of $\delta$ between time $t_{k-1}$ and $t_{k}$, the state $\mathbf{x}\left(t_{k}\right)$ is related to the previous state $\mathbf{x}\left(t_{k-1}\right)$ with

$$
\mathbf{x}\left(t_{k}\right)=\mathbf{F x}\left(t_{k-1}\right)
$$

where

$$
\mathbf{F}=\left[\begin{array}{cc}
\mathbf{1} & \Delta t \cdot \mathbf{1} \\
\mathbf{0} & \mathbf{1}
\end{array}\right]
$$

where 1 is the identity matrix and $\Delta t$ is the time between $t_{k-1}$ and $t_{k}$. Further, the observations, $b\left(t_{k}\right)$ at time $t_{k}$ can then be related to the state using

$$
\mathbf{b}\left(t_{k}\right)=\mathbf{H x}\left(t_{k}\right)+\mathbf{w}\left(t_{k}\right)
$$

where $\mathbf{w}\left(t_{k}\right)$ is the noise and

$$
\mathbf{H}=\left[\begin{array}{ll}
G & 0
\end{array}\right]
$$


where $G$ is given by (5). The Kalman filter uses the previous state $\mathbf{x}\left(t_{k-1}\right)$ and the observations $b\left(t_{k}\right)$ to estimate the current state $\mathbf{x}\left(t_{k}\right)$. This is given by

$$
\mathbf{x}\left(t_{k}\right)=\left(\mathbf{1}-\mathbf{K}\left(t_{k}\right) \mathbf{H}\right) \mathbf{F} \mathbf{x}\left(t_{k-1}\right)+\mathbf{K}\left(t_{k}\right) b\left(t_{k}\right)
$$

where the Kalman gain $\mathbf{K}\left(t_{k}\right)$ is given by (23), shown at the bottom of the page, where $\mathbf{R}\left(t_{k}\right)$ and $\mathbf{Q}\left(t_{k-1}\right)$ are the measurement- and system covariance matrices. If these matrices are known or are possible to estimate they are used in the Kalman filter to weight the signals. The system covariance matrix $\mathbf{Q}\left(t_{k}\right)$ contains the information about the uncertainties affecting the estimated state $\mathbf{x}\left(t_{k}\right)$. In an implementation of the measurement system this would, in addition to errors induced in the updating process, also include error sources related to the AUT. These could be both electrical, such as crosstalk and limited accuracy in the timing between front-ends, and mechanical, such as variations in the position of the AUT (e.g., due to wind).

The observation covariance matrix, $\mathbf{R}\left(t_{k}\right)$, includes information about uncertainties related to the observations. These are mainly caused by error sources affecting the probes. Apart from electrical and mechanical errors similar to the ones affecting the AUT, the main error is the thermal noise on the observation. There may also be a polarization error due to non-ideal probes.

All the uncertainties discussed here will have the effect of an amplitude and phase-shift of the far-field pattern of the AUT. The estimated far-field pattern of a given antenna element will thus include all other error sources in addition to the effect of snowfall, which is the desired information.

\section{NUMERICAL RESULTS}

In this section, the performance of an implementation of the method described above is assessed using numerical data from simulations using the Numerical Electromagnetics Code (NEC) [15]. In the simulations, all effects due to mutual coupling between nearby antenna elements (e.g., scan blindness) have been neglected. These effects can be taken into account when performing the measurements by using additional basis functions and careful selection of the probes used at a given time.

\section{A. System Setup}

The antenna array considered here is similar to the EISCAT_3D test array outside Kiruna, Sweden. The antenna elements are the same crossed Yagi-antennas as the ones analyzed in [1], these are also shown in Fig. 1. The measurement system is designed with the final radar system in mind, where the size of the antenna array could be on the order of 125-by-125 m. The antenna elements are mounted at

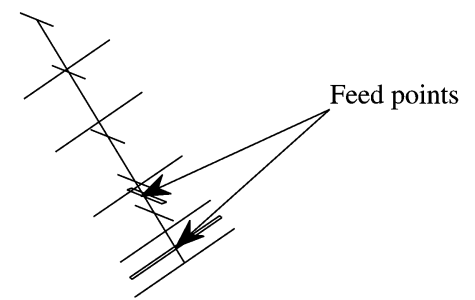

(a)

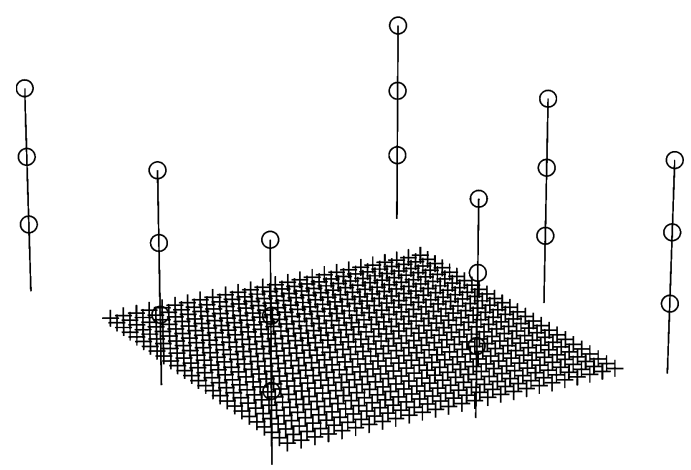

(b)

Fig. 1. The proposed measurement system setup (b) and antenna element (a). The number of antenna elements plotted is in the figure for clarity lower than in the actual system. The probes are in (b) denoted by ' $O$ '.

an elevation angle of $55^{\circ}$ and also rotated $45^{\circ}$ with respect to its own axis. It should be noted that this system setup is only considered in order to define realistic locations of the probes. It is thus not designed with any array parameters in mind.

The probes are assumed to be mounted on $150 \mathrm{~m}$ high towers which are spread out around the antenna array. Although there may be some interference due to reflections from the towers this effect should be small since there are no towers in the main beam direction of the antenna array. There are three probes on each tower at 50, 100, and $150 \mathrm{~m}$. This setup is shown in Fig. 1.

Fig. 2 shows the simulated far-field (solid line) of one antenna element and the far-field estimated using the equivalent electric current method (dotted line) for the element in the middle of the array in Fig. 1. This element is assumed to be used as the reference element and it is therefore temperature controlled and free from snow. The reference is the results from a full NEC-2 simulation while, for the estimation, only the field calculated at the probe locations was used. It can be seen that the results for both the amplitude and phase is accurate for the considered element. Only the results for one polarization is shown (the lower of the ones shown in Fig. 1(a).

Since the position of the probes relative to the AUT will be different for different antenna elements in the array, the error introduced in Fig. 2 could vary between the antenna elements.

$$
\begin{aligned}
\mathbf{K}\left(t_{k}\right)=\mathbf{P}\left(t_{k} \mid t_{k-1}\right) \mathbf{H}^{H} \mathbf{S}^{-1}\left(t_{k}\right) & \text { Kalman gain } \\
\mathbf{S}\left(t_{k}\right)=\mathbf{H} \mathbf{P}\left(t_{k} \mid t_{k-1}\right) \mathbf{H}^{H}+\mathbf{R}\left(t_{k}\right) & \text { residual covariance } \\
\mathbf{P}\left(t_{k} \mid t_{k-1}\right)=\mathbf{F}\left(t_{k}\right) \mathbf{P}\left(t_{k-1} \mid t_{k-1}\right) \mathbf{F}^{H}+\mathbf{Q}\left(t_{k-1}\right) & \text { predicted estimate covariance } \\
\mathbf{P}\left(t_{k} \mid t_{k}\right)=\left(\mathbf{1}-\mathbf{K}\left(t_{k}\right) \mathbf{H}\right) \mathbf{P}\left(t_{k} \mid t_{k-1}\right) & \text { updated estimate covariance. }
\end{aligned}
$$




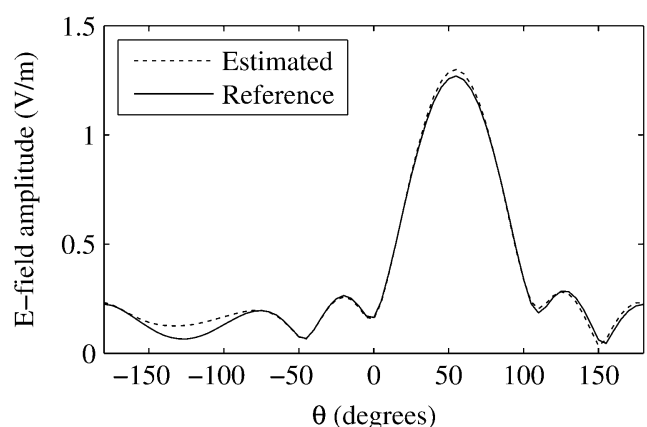

(a)

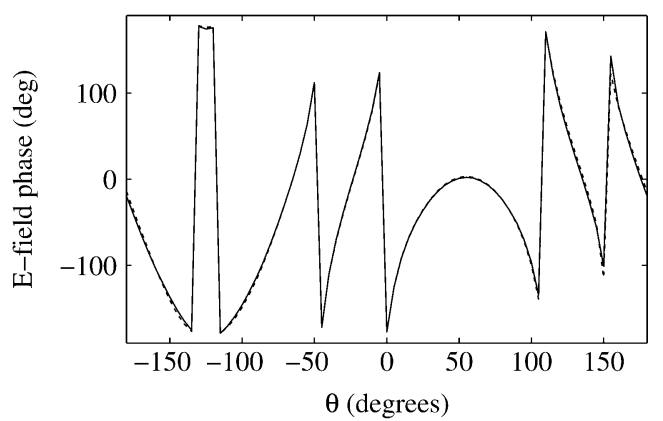

(b)

Fig. 2. The far-field amplitude (a) and phase (b) for the antenna element in middle of the array. The probes are assumed to be noise free.

This is shown in Fig. 3 for the main beam direction of the antenna element (along the y-axis with an elevation angle of $55^{\circ}$. The error is significantly larger in the middle, front part of the array. This is because there are no probes in the main beam of these antenna elements. This numerical error can be reduced when implementing the methods as discussed previously (e.g., using the CG method to solve the normal equations if the LS estimator is used). The main interest here is however to compare the LS estimator with the Kalman filter during noisy conditions. For this reason, the simulations are performed for an element in the middle of the array where the numerical errors in this case is small.

\section{B. Modeling Snow Covered Wire Antennas}

The snow is in this paper modeled as a dielectric medium with a complex permittivity using a model proposed in [16]. The relative permittivity, $\varepsilon_{s}$, of snow is in the model given by

$$
\operatorname{Re}\left\{\varepsilon_{s}\right\}=1+1.7 \rho_{d}+0.7 \rho_{d}^{2}+8.7 W+70 W^{2}
$$

where $\rho_{d}$ is the relative density of snow compared to water, and $W$ is the water content by volume. For the imaginary part, only the frequency and water content is of importance, and this is given by

$$
\operatorname{Im}\left\{\varepsilon_{s}\right\}=\frac{\omega}{2 \pi 10^{9}}\left(0.9 W+7.5 W^{2}\right) .
$$

The imaginary part of the relative permittivity is often neglected but is included here for completeness. This model is empirical and only the effects of the density of dry snow, the water content, and the frequency are included even though the effect of both temperature and pollutants may be added to the model if

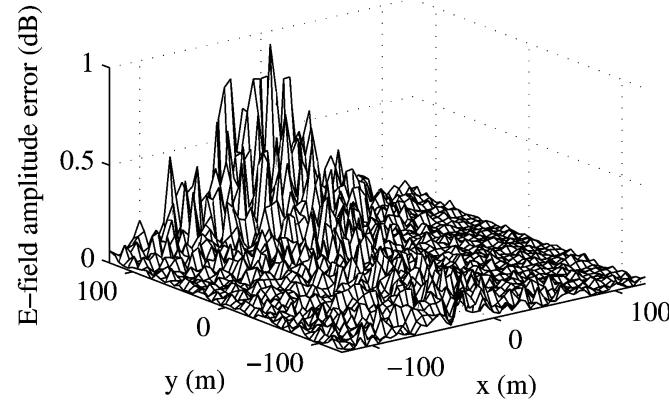

(a)

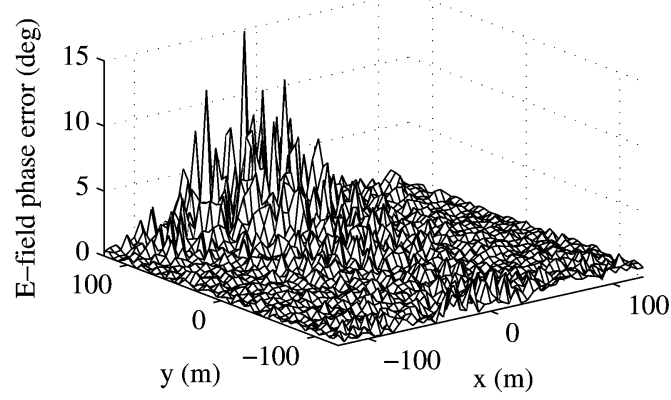

(b)

Fig. 3. The estimated far-field amplitude (a) and phase (b) error in the direction of the main beam of the antenna elements for different positions in the array.

desired. Also, numerous other models have been proposed, see, e.g., [17]-[19].

Since the parameters affecting the permittivity of snow can vary quickly with changing conditions it is not feasible to take every possible situation into account. Instead a worst case scenario was adopted. The water content is assumed to be $10 \%$ by volume and the density $600 \mathrm{~kg} / \mathrm{m}^{3}$. This corresponds to wet and heavy snow, which have the highest effect on the antennas. The real part of the relative permittivity is in this case 3.7 while the imaginary part is 0.037 . The snow-covered antenna elements are modeled in the same way as insulated wire antennas. This can be done by a modification of the impedance matrix as described in [20].

\section{Performance During Snowfall Under Noise-Free Conditions}

Simulations were done for a test case to compare the performance of the LS estimator method with the Kalman filter approach. These simulations were done for one antenna element located in the left, back corner of the antenna array shown in Fig. 1 with the antenna element located in the middle of the array used as the reference element. In this simulation the signal is assumed to be noise-free. The Kalman filter here gave identical results as the LS estimator.

The test scenario that is used both here, in the noise-free, and in the noisy conditions considered later is shown in Fig. 4. At time $\mathrm{T}=10$, there is the onset of the snowfall. The thickness of the snow layer increases gradually until there is a $0.5 \mathrm{~mm}$ thick layer of snow covering the antennas. In the simulations, the snow covers all wires of the antennas completely. This will most often not be the case in reality where the snow will cover only the top part of the wires. The simulation is divided into 


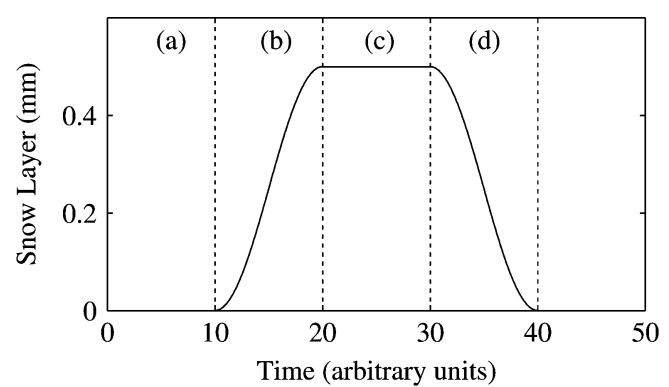

Fig. 4. The thickness of the snow layer as a function of time. The letters (a) to (d) denotes periods used for the analysis of the results.

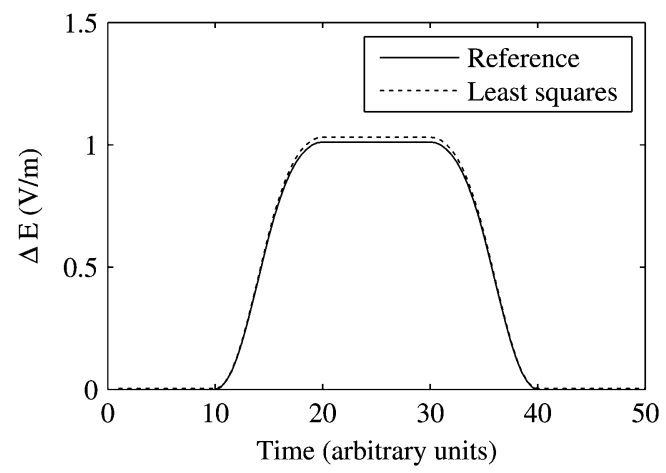

Fig. 5. Performance of the least squares solution during snowfall.

four different periods in order to evaluate the performance of the measurement system under different dynamic situations.

In Fig. 5 the amplitude of the difference between reference antenna's and the AUT's far-field is shown. The estimated farfield difference here gives accurate results. There is a small bias during the period when there is a constant snow layer on the antennas. This bias is, however, well within the acceptable limits of the system.

\section{Performance During Snowfall Under Noisy Conditions}

In the simulations considered here, the signal transmitted by the probes is assumed to be noisy. The noise is assumed to have a gaussian distribution with zero mean. Since the amplitude of the signal received by the antenna elements will depend on both the distance to the probes and the antenna gain in the direction of the probes the signal-to-noise ratio (SNR) will vary significantly between different probes. The SNR for the AUT considered in this section varies from less than $-7 \mathrm{~dB}$ to over $32 \mathrm{~dB}$ for different probes.

To evaluate the performance, the complex error in the estimated far-field was calculated. This can be seen in Fig. 6 where four different periods were analyzed. The letters (a), (b), (c), and (d) in the Fig. 6 are denoted by the same letters in Fig. 4. To create these plots, a total of 100 simulations were used. The numerical values are also shown in Table I, where the amplitude of the mean error is used to compare the systematic errors induced by the estimation methods. The standard deviation is used as a metric of the precision. During all periods, the Kalman filter gives more precise results than the least squares solution, which is the result of the averaging over time. During period (c) and (d)

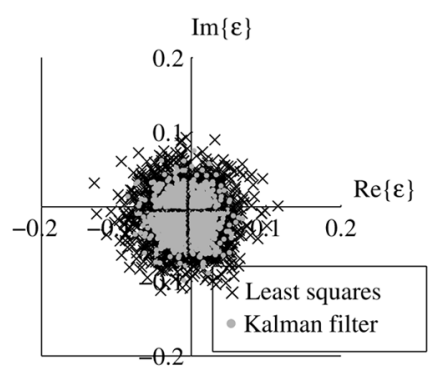

(a)

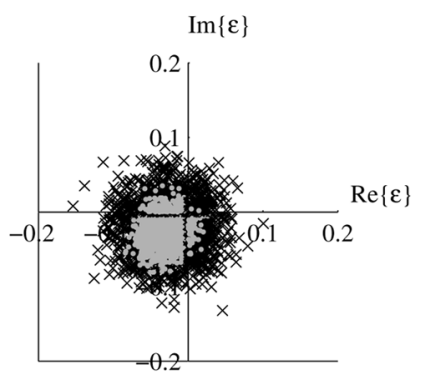

(c)

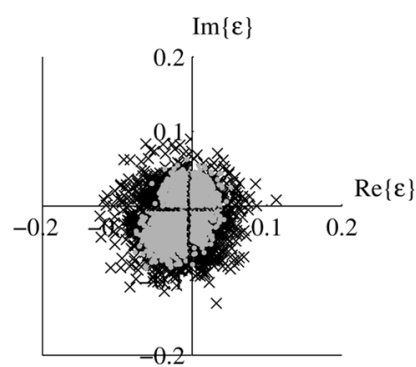

(b)

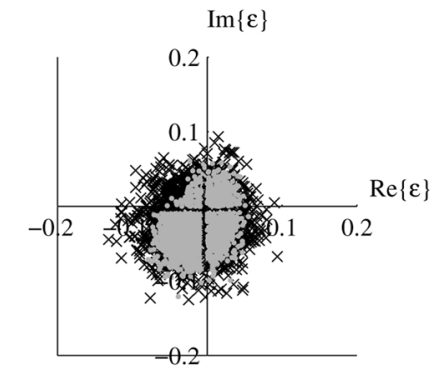

(d)
Fig. 6. The complex error induced due to the noise on the test signal ( $\varepsilon$ denotes the error). The four subplots (a) to (d) refer to the corresponding periods shown in Fig. 4. The units are V/m.

TABLE I

ACCURACY OF THE ESTIMATED ELECTRIC FIELD DIFFERENCE

\begin{tabular}{l|c|c}
\hline \multicolumn{3}{c}{ Standard deviation } \\
\hline Period & Least squares & Kalman Filter \\
(a) & $0.047 \mathrm{~V} / \mathrm{m}$ & $0.030 \mathrm{~V} / \mathrm{m}$ \\
(b) & $0.047 \mathrm{~V} / \mathrm{m}$ & $0.036 \mathrm{~V} / \mathrm{m}$ \\
(c) & $0.047 \mathrm{~V} / \mathrm{m}$ & $0.024 \mathrm{~V} / \mathrm{m}$ \\
(d) & $0.047 \mathrm{~V} / \mathrm{m}$ & $0.040 \mathrm{~V} / \mathrm{m}$ \\
\hline \hline \multicolumn{3}{c}{ Amplitude of mean error } \\
\hline Period & Least squares & Kalman Filter \\
(a) & $0.016 \mathrm{~V} / \mathrm{m}$ & $0.016 \mathrm{~V} / \mathrm{m}$ \\
(b) & $0.024 \mathrm{~V} / \mathrm{m}$ & $0.018 \mathrm{~V} / \mathrm{m}$ \\
(c) & $0.032 \mathrm{~V} / \mathrm{m}$ & $0.038 \mathrm{~V} / \mathrm{m}$ \\
(d) & $0.023 \mathrm{~V} / \mathrm{m}$ & $0.031 \mathrm{~V} / \mathrm{m}$ \\
\hline
\end{tabular}

there is, however, a larger systematic error in the Kalman filter while the bias in the LS estimator is slightly larger in period (b). The difference in the amplitude of the mean error is statistically significant for periods (b), (c), and (d).

\section{CONCLUSION}

The measurement system presented in this paper uses the equivalent electric current method to estimate the far-field of an antenna element in a physically large antenna array. The method enables continuous measurements of the performance of all antenna elements in array without the need of locating any probes in the main beam of the antenna array. Also, using the difference of the far-field between two elements it is possible to incorporate far-field sources, which may be at an unknown distance from the antenna element, into the system of equations and retain the phase information. The simulations shows that, in some cases, the Kalman filter gives a larger systematic error than the LS estimator. The LS estimator might therefore be more suitable in spite of its larger random variations. On the other hand, a Kalman filter can be used to estimate the state of the antenna array in more general terms, where calibration systems further 
down the receiver chain may be included. This is a topic that should be studied further.

Although only one test scenario has been considered the results from the simulations shows that by using this type of measurement system, it is possible to improve the knowledge about the far-field characteristics of an antenna. This means that the availability of the radar system and the quality of the scientific measurements can be increased.

\section{REFERENCES}

[1] T. Lindgren and J. Ekman, "Performance of a Yagi antenna during snowfall," presented at the Int. Symp. on Antennas and Propagation, Taipei, Taiwan, Oct. 2008.

[2] P. Petre and T. K. Sarkar, "Planar near-field to far-field transformation using an equivalent magnetic current approach," IEEE Trans. Antennas Propag., vol. 40, no. 11, pp. 1348-1356, Nov. 1992.

[3] T. K. Sarkar and A. Taaghol, "Near-field to near/far-field transformation for arbitrary near-field geometry utilizing an equivalent electric current approach," IEEE Trans. Antennas Propag., vol. 47, no. 3, pp. 566-573, Mar. 1999.

[4] G. Wannberg, EISCAT_3D Design Specification Document EISCAT Scientific Association, Tech. Rep., 2005 [Online]. Available: https://e7. eiscat.se/groups/EISCAT_3D_info/P_S_D_7.pdf

[5] G. Stenberg, J. Borg, J. Johansson, and G. Wannberg, "Simulation of post-ADC digital beam-forming for large area radar receiver arrays," presented at the IEEE Int. RF and Microwave Conf. Putra Jaya, Malaysia, Sep. 12-14, 2006.

[6] G. Stenberg, T. Lindgren, and J. Johansson, "A picosecond accuracy timing system based on L1-only GNSS receivers for a large aperture array radar," presented at the ION GNSS Savannah, GA, Sep. 16-19, 2008.

[7] G. Wannberg, I. Wolf, L.-G. Vanhainen, K. Koskenniemi, J. Röttger, M. Postila, J. Markkanen, R. Jacobsen, A. Stenberg, R. Larsen, S. Eliassen, S. Heck, and A. Huuskonen, "The EISCAT svalbard radar: A case study in modern incoherent scatter radar system design," Radio Sci., vol. 32, no. 6, pp. 2283-2307, Nov.-Dec. 1997.

[8] P. G. Smith, "Measurement of the complete far-field pattern of large antennas by radio-star sources," IEEE Trans. Antennas Propag., vol. 14, no. 1, pp. 6-16, Jan. 1966.

[9] P. Talaga, "The measurement of a large antenna using a spacecraft as a receiver," IEEE Trans. Antennas Propag., vol. 38, no. 6, pp. 883-888, Jun. 1990.

[10] A. D. Yaghian, "An overview of near-field antenna measurements," IEEE Trans. Antennas Propag., vol. 34, no. 1, Jan. 1986.

[11] D. C. Law, J. R. Khorrami, W. B. Sessions, and M. K. Shanahan, "Radiation patterns of a large UHF phased-array antenna: A comparison of measurements using satellite repeaters and patterns derived from measurements of antenna current distributions," IEEE Antennas Propag. Mag., vol. 39, no. 5, pp. 88-93, Oct. 1997.

[12] P.-S. Kildal, Foundations of Antennas, a Unified Approach. Lund, Sweden: Studentlitteratur, 2000.

[13] T. K. Sarkar and E. Arvas, "On a class of finite step iterative methods (conjugate directions) for the solution of an operator equation arising in electromagnetics," IEEE Trans. Antennas Propag., vol. 33, no. 10, Oct. 1985.
[14] S. M. Kay, Fundamentals of Statistical Signal Processing: Estimation Theory. Englewood Cliffs, NJ: Prentice Hall, 1993.

[15] G. Burke and A. Poggio, Numerical Electromagnetics Code Method of Moments Lawrence Livermore Nat. Lab., Livermore, CA, Rep. UCID18834, 1981.

[16] M. Tiuri, A. Sihvola, E. Nyfors, and M. Hallikaiken, "The complex dielectric constant of snow at microwave frequencies," IEEE J. Oceanic Eng., vol. 9, no. 5, pp. 377-382, Dec. 1984.

[17] D. A. Boyarskii and V. V. Tikhonov, "Microwave effective permittivity model of media of dielectric particles and applications to dry and wet snow," in Proc. Int. Geoscience and Remote Sensing Symp., Aug. 1994, vol. 4, pp. 2065-2067.

[18] C. Mätzler, "Microwave permittivity of dry snow," IEEE Trans. Geosci. Remote Sens., vol. 34, no. 2, pp. 573-581, Mar. 1996.

[19] A. D. Frolov and Y. Y. Macheret, "On dielectric properties of dry and wet snow," Hydrol. Process., vol. 13, pp. 1755-1760, 1999.

[20] J. H. Richmond and E. H. Newman, "Dielectric coated wire antennas," Radio Sci., vol. 11, no. 1, pp. 13-20, Jan. 1976.

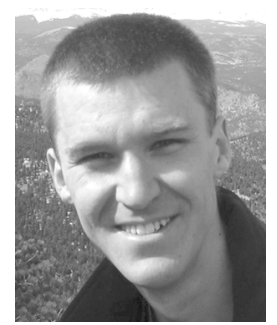

Tore Lindgren (M'09) received the Ph.D. degree in electrical engineering from Luleå University of Technology, Luleå, Sweden, in 2009.

He is currently working as a Researcher at Luleå University of Technology. His research interest is in antenna array measurement techniques for radar and GNSS receiver applications, and simulation of radiofrequency identification systems.

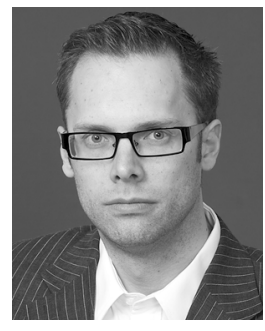

Jonas Ekman (M'06) was born in Boden, Sweden, in 1972. He received the Ph.D. degree in electrical engineering from Luleå University of Technology, Luleå, Sweden, in 2003.

From 2003 to 2007, he was working as a Researcher at Luleå University of Technology. During 2005 and 2006, he did his Postdoctoral research in full-wave, time domain, PEEC modeling at the EMC Laboratory, University of L'Aquila, Italy. In 2008, he was appointed Associate Professor at Luleå University of Technology. His research interests are in computational electromagnetics, in particular, the use of the PEEC method for realistic electromagnetic modeling.

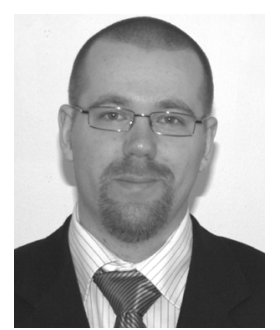

Staffan Backén (S'10) received the M.Sc. degree in electrical engineering from Luleå University of Technology, Luleå, Sweden, in 2004 and the Licentiate degree in electrical engineering from Luleå University of Technology, Luleå, in 2007, where he is currently working toward the $\mathrm{Ph} . \mathrm{D}$. degree.

His research focus is on GNSS array processing and receiver architecture. 